\title{
Creatine Kinase BB to Total Creatine Kinase Ratio Measurement
}

National Cancer Institute

\section{Source}

National Cancer Institute. Creatine Kinase BB to Total Creatine Kinase Ratio

Measurement. NCI Thesaurus. Code C79466.

The determination of the ratio of creatine kinase BB compared to total creatine kinase present in a sample. The measurement may be expressed as a ratio or percentage. 